\title{
Increased Expression of Herpes Virus-Encoded hsv1-miR-H18 and hsv2-miR-H9-5p in Cancer-Containing Prostate Tissue Compared to That in Benign Prostate Hyperplasia Tissue
}

\author{
Seok Joong Yun ${ }^{1, *}$, Pildu Jeong ${ }^{1, *}$, Ho Won Kang ${ }^{1}$, Helen Ki Shinn ${ }^{2}$, Ye-Hwan Kim ${ }^{1}$, Chunri Yan ${ }^{1}$, Young-Ki Choi ${ }^{3}$, \\ Dongho Kim ${ }^{4}$, Dong Hee Ryu ${ }^{5}$, Yun-Sok Ha ${ }^{6}$, Tae-Hwan Kim ${ }^{6}$ Tae Gyun Kwon ${ }^{6}$, Jung Min Kim ${ }^{7}$ Sang Heon Suh ${ }^{8}$,

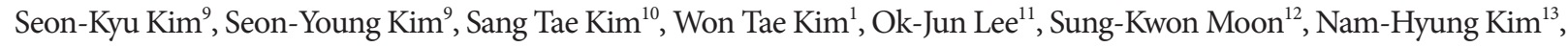 \\ Isaac Yi Kim ${ }^{14}$, Jayoung $\mathrm{Kim}^{15,16,17}$, Hee-Jae Cha ${ }^{18}$, Yung-Hyun $\mathrm{Choi}^{19}$, Eun-Jong $\mathrm{Cha}^{20}$, Wun-Jae Kim ${ }^{1}$
}

${ }^{1}$ Department of Urology, Chungbuk National University College of Medicine, Cheongju, Korea

${ }^{2}$ Department of Anesthesiology and Pain Medicine, Inha University College of Medicine, Incheon, Korea

${ }^{3}$ Department of Microbiology, College of Medicine and Medical Research Institute, Chungbuk National University, Cheongju, Korea

${ }^{4}$ Bio Medical Laboratories, Daejeon, Korea

${ }^{5}$ Department of Surgery, Chungbuk National University, College of Medicine and Medical Research Institute, Cheongju, Korea

${ }^{6}$ Department of Urology, Kyungpook National University School of Medicine, Daegu, Korea

${ }^{7}$ NAR Center Inc., Daejeon Oriental Hospital of Daejeon University, Daejeon, Korea

${ }^{8}$ The Laboratory of Vascular Biology and Stem Cells, BioMedical Research Center, Graduate School of Medical Science and Engineering, Korea Advanced Institute of Science and Technology (KAIST), Daejeon, Korea

${ }^{9}$ Medical Genomics Research Center, Korean Bioinformation Center, Korea Research Institute of Bioscience and Biotechnology, Department of Functional Genomics, University of Science and Technology, Daejeon, Korea

${ }^{10}$ Biomedical Research Institute, Department of Neuropsychiatry, Seoul National University Bundang Hospital, Seoul National University College of Medicine, Seoul, Korea

${ }^{11}$ Department of Pathology, Chungbuk National University College of Medicine, Cheongju, Korea

${ }^{12}$ School of Food Science and Technology, Chung-Ang University, Anseong, Korea

${ }^{13}$ Department of Animal Sciences, Chungbuk National University, Cheongju, Korea

${ }^{14}$ The Section of Urologic Oncology and Dean and Betty Gallo Prostate Cancer Center, The Cancer Institute of New Jersey, UMDNJ-Robert Wood Johnson Medical School, New Brunswick, NJ, USA

${ }^{15}$ Department of Surgery, Harvard Medical School, Boston, MA, USA

${ }^{16}$ Division of Cancer Biology and Therapeutics, Departments of Surgery and Biomedical Sciences, Samuel Oschin Comprehensive Cancer Institute, CedarsSinai Medical Center, Los Angeles, CA, USA

${ }^{17}$ Department of Medicine, University of California Los Angeles, Los Angeles, CA, USA

${ }^{18}$ Department of Parasitology and Genetics, Kosin University College of Medicine, Busan, Korea

${ }^{19}$ Department of Biochemistry, Dongeui University College of Oriental Medicine, Busan, Korea

${ }^{20}$ Department of Biomedical Engineering, Chungbuk National University, Cheongju, Korea

\footnotetext{
Corresponding author: Wun-Jae Kim (iD http://orcid.org/0000-0002-8060-8926 Department of Urology, Chungbuk National University College of Medicine, 1 Chungdae-ro, Seowon-gu, Cheongju 28644, Korea E-mail: wjkim@chungbuk.ac.kr / Tel: +82-43-269-6371 / Fax: +82-43-269-6144 *Seok Joong Yun and Pildu Jeong contributed equally to this study as co-first authors.

Submitted: February 26, 2016 / Accepted after revision: April 17, 2016
}

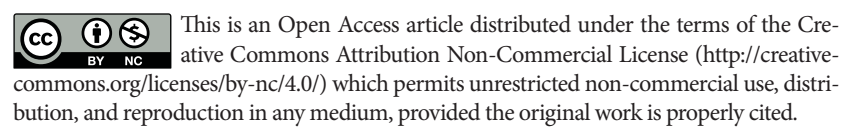
bution, and reproduction in any medium, provided the original work is properly cited. 
Purpose: Previously, we reported the presence of virus-encoded microRNAs (miRNAs) in the urine of prostate cancer (CaP) patients. In this study, we investigated the expression of two herpes virus-encoded miRNAs in prostate tissue.

Methods: A total of 175 tissue samples from noncancerous benign prostatic hyperplasia (BPH), 248 tissue samples from patients with $\mathrm{CaP}$ and $\mathrm{BPH}$, and 50 samples from noncancerous surrounding tissues from these same patients were analyzed for the expression of two herpes virus-encoded miRNAs by real-time polymerase chain reaction (PCR) and immunocytochemistry using nanoparticles as molecular beacons.

Results: Real-time reverse transcription-PCR results revealed significantly higher expression of hsv1-miR-H18 and hsv2-miR$\mathrm{H} 9-5 \mathrm{p}$ in surrounding noncancerous and $\mathrm{CaP}$ tissues than that in $\mathrm{BPH}$ tissue (each comparison, $\mathrm{P}<0.001$ ). Of note, these miRNA were expressed equivalently in the $\mathrm{CaP}$ tissues and surrounding noncancerous tissues. Moreover, immunocytochemistry clearly demonstrated a significant enrichment of both hsv1-miR-H18 and hsv2-miR-H9 beacon-labeled cells in CaP and surrounding noncancerous tissue compared to that in BPH tissue (each comparison, $\mathrm{P}<0.05$ for hsv1-miR-H18 and hsv2miR-H9).

Conclusions: These results suggest that increased expression of hsv1-miR-H18 and hsv2-miR-H95p might be associated with tumorigenesis in the prostate. Further studies will be required to elucidate the role of these miRNAs with respect to CaP and herpes viral infections.

Keywords: MicroRNAs; Prostate Neoplasms; Herpesviridae; Prostate Hyperplasia

- Fund/Grant Support: This research was supported by the Functional Districts of the Science Belt support program (No. 2015K000284), and the National Research Foundation of Korea (No. NRF-2014R1A2A1A09006983, NRF-2014R1A2A2A04007036, and NRF-2015R1A2A2A03004100) of Ministry of Science, ICT and Future Planning.

- Research Ethics: The collection and analysis of all samples was approved by the Institutional Review Board of Chungbuk National University, and written informed consent was obtained from each subject (approval numbers: 2006-01-001 and GR2010-12-010).

- Conflict of Interest: No potential conflict of interest relevant to this article was reported.

\section{INTRODUCTION}

Prostate cancer $(\mathrm{CaP})$ is one of the most common malignancies in Western males, and the prevalence of $\mathrm{CaP}$ in Korean men is increasing [1,2]. Prostate infection and inflammation caused by bacteria and viruses are thought to be important drivers of carcinogenesis in $\mathrm{CaP}$ [3-5]. A meta-analysis investigating the relationship between sexually transmitted diseases (STDs) and the risk for CaP suggested that men with a history of infection with any STD or gonorrhea, specifically, exhibited increased risks of $\mathrm{CaP}$ (1.5 fold and 1.2 fold, respectively) [6]. However, the existence of a causal relation between inflammation from infection and prostate carcinogenesis is still debatable [3]. Several studies have suggested that viral microRNAs (miRNAs), mostly derived from herpes viruses, facilitate cross-talk between the virus and host during viral infection and pathogenesis $[7,8]$. For instance, viral miRNAs acting directly as oncogenes can be illustrated by the Kaposi's sarcoma-associated her- pesvirus miR-K12-10 and miR-K12-12, both encoded within the transcript of the A isoform of kaposin, which is known to mediate cellular transformation through loss of growth control and cell surface-mediated inhibition [9]. Thus, a causal association between viral miRNAs and carcinogenesis should not be dismissed, even though evidence is limited.

Recently, we reported the presence of virus-encoded miRNAs in the urine of patients with $\mathrm{CaP}$ as a marker to distinguish between cancer and noncancerous benign prostatic hyperplasia (BPH) [10]. Urinary hsv2-miR-H9-5p proved to be a better diagnostic than serum prostate-specific antigen (PSA) in the indeterminate "gray zone," and it was comparable to serum PSA in patients with $\mathrm{CaP}$ who underwent transrectal biopsy. The combination of miRNA and serum PSA measurements, therefore, may prevent unnecessary biopsy, making miRNA a clinically valuable diagnostic marker. To further this line of inquiry and potentially identify an additional marker of $\mathrm{CaP}$, the expression of two viral miRNAs, hsv1-miR-H18 and hsv2-miR- 
H9-5p, was compared in BPH tissue, cancer tissue, and surrounding noncancerous tissue, and their association with pathological outcomes was investigated.

\section{MATERIALS AND METHODS}

\section{Cases and Controls}

For this study, a total of 175 samples from noncancerous BPH tissues, 50 samples from noncancerous surrounding tissues, and 248 samples from cancerous tissues were obtained from patients with $\mathrm{CaP}$ and $\mathrm{BPH}$ at Chungbuk National University Hospital (Table 1). A schematic of the study design, which included three different cohorts, is shown in Fig. 1. All tissues were harvested from patients with $\mathrm{CaP}$ who underwent radical prostatectomy or palliative transurethral resection (TUR), or patients with BPH who underwent TUR. Tissue samples were macrodissected within 15 minutes of surgical resection. Each prostate specimen was confirmed by analysis of fresh-frozen sections, and the remaining tissue was frozen in liquid nitrogen and stored at $-80^{\circ} \mathrm{C}$ until use. BPH controls with serum PSA levels $\geq 3 \mathrm{ng} / \mathrm{mL}$ underwent transrectal prostate biopsy prior to TUR to rule out the presence of cancer. Patients receiving neoadjuvant therapies, such as androgen deprivation or radiation therapy, were excluded from the study. Gleason grades were assigned to specimens obtained from 12-core transrectal biopsies, transurethral resection of the prostate or radical prostatectomy.

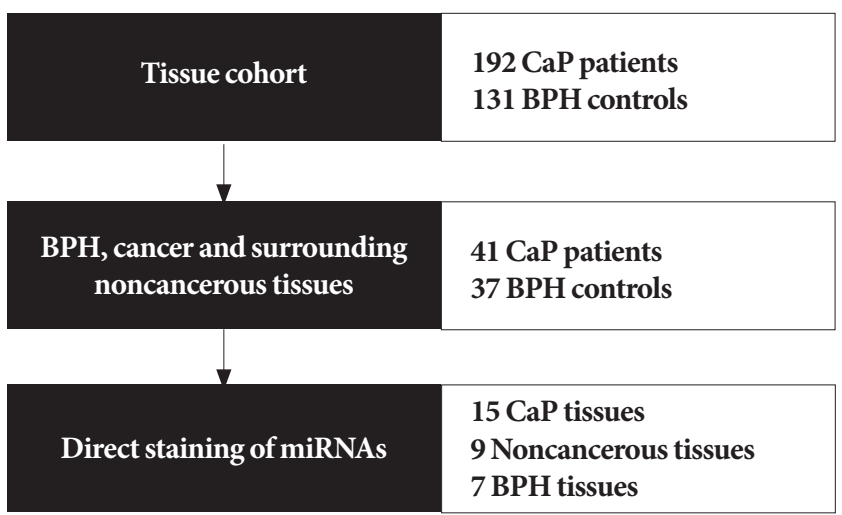

Fig. 1. Overview of the study design. Expression of hsv1-miRH18 and hsv2-miR-H9-5p was assessed in 192 prostate cancer $(\mathrm{CaP})$ tissues and 131 benign prostatic hyperplasia $(\mathrm{BPH})$ noncancerous tissues. Then, expression of these microRNAs (miRNAs) was compared between matched cancerous and surrounding noncancerous tissues from 41 patients, as well as in noncancerous tissues from BPH patients. Lastly, the 2 miRNAs were directly stained in cancerous tissues, surrounding noncancerous tissues, and BPH tissues.

Table 1. Clinical characteristics of the patients and controls

\begin{tabular}{|c|c|c|c|c|c|c|}
\hline \multirow{2}{*}{ Characteristic } & \multicolumn{2}{|c|}{$\mathrm{BPH}$ and prostate cancer tissues } & \multicolumn{2}{|c|}{$\begin{array}{l}\mathrm{BPH}, \text { surrounding noncancerous, and } \\
\text { prostate cancer tissues }\end{array}$} & \multicolumn{2}{|c|}{ Direct staining } \\
\hline & Control & Case & Control & Case & Control & Case \\
\hline No. & 131 & 192 & 37 & 41 & 7 & 24 \\
\hline Age (yr) & $71.5(46-89)$ & $69.1(50-92)$ & $71.6(58-34)$ & $69.2(58-80)$ & $73.6(68-80)$ & $68.9(65-73)$ \\
\hline PSA (ng/mL) & $4.9 \pm 9.4$ & $143.0 \pm 584.5$ & $4.3 \pm 4.9$ & $14.9 \pm 15.9$ & $11.7 \pm 9.0$ & $37.3 \pm 87.2$ \\
\hline \multicolumn{7}{|l|}{ Operation (\%) } \\
\hline TURP & $131(100)$ & $57(29.7)$ & $37(100)$ & - & $7(100)$ & - \\
\hline Radical prostatectomy & - & $135(70.3)$ & - & $41(100)$ & - & $24(100)$ \\
\hline \multicolumn{7}{|l|}{ Gleason score (\%) } \\
\hline$\leq 6$ & - & $7(3.6)$ & - & $6(14.6)$ & - & $0(0)$ \\
\hline 7 & - & $96(50.0)$ & - & $24(58.5)$ & - & $15(62.5)$ \\
\hline 8 & - & $34(17.7)$ & - & $7(17.0)$ & - & $3(12.5)$ \\
\hline 9 & - & $49(25.5)$ & - & $3(7.3)$ & - & $6(25)$ \\
\hline 10 & - & $6(3.1)$ & - & $1(2.4)$ & - & $0(0)$ \\
\hline \multicolumn{7}{|l|}{ TNM stage } \\
\hline $\mathrm{T} 3$ or less & & $126(65.6)$ & & $41(100)$ & & $21(87.5)$ \\
\hline $\mathrm{T} 4$ or metastatic & & $66(34.4)$ & & - & & $3(12.5)$ \\
\hline
\end{tabular}

Values are presented as median (range), mean \pm standard deviation, or number (\%).

$\mathrm{BPH}$, benign prostatic hyperplasia; PSA, prostate-specific antigen; TURP, transurethral resection of the prostate. 
Tumor stage was estimated from specimens obtained by radical prostatectomy, or from computed tomography, magnetic resonance imaging, or bone scans. The collection and analysis of all samples was approved by the Institutional Review Board of Chungbuk National University, and written informed consent was obtained from each subject (approval numbers: 2006-01001 and GR2010-12-010). Gleason grades and TNM 2002 staging were used as prognostic factors.

\section{Purification of miRNA}

Total miRNAs were isolated from $1 \mathrm{mg}$ of each tissue specimen using the Genolution miRNA Purification Kit (Genolution Pharmaceuticals Inc., Seoul, Korea). Reverse transcription of miRNA was conducted with the miScript Reverse Transcription Kit (Qiagen Korea, Seoul, Korea) according to the manufacturer's recommended protocol.

\section{Amplification of miRNA by Real-Time PCR}

To quantify miRNA expression, real-time polymerase chain reaction (PCR) amplification was performed using a Rotor-Gene Q (Qiagen, Valencia, CA, USA) and the miScript PCR Starter Kit (Qiagen Korea). Chemically synthesized RNA oligonucleotides (Cosmogenetech, Seoul, Korea) corresponding to the target miRNAs were used to generate standard curves. The standard curves ranged from 30 to $3 \times 10^{4}$ copies. The target miRNAs (hsv1-miR-H18 and hsv2-miR-H9-5p) were amplified using primers CCCGCCCGCCGGACGCCGGGACC and CTCGGAGGTGGAGTCGCGGT, respectively. The conditions used for real-time PCR are described in the manufacturer's protocol. All samples were tested in triplicate.

Preparation of $\mathrm{QD}_{565} / \mathrm{QD}_{525}$ Nanoparticle-Conjugated hsv1miR-H18 and hsv2-miR-H9-Responsive Molecular Beacons

$\mathrm{QD}_{565}$-hsv1-miR-H18 and $\mathrm{QD}_{565}$-hsv2-miR-H9 beacons specific for hsv1-miR-H18 and hsv2-miR-H9 were prepared as previously described [11]. The sequences of the hsv1-miR-H18 and hsv2-miR-H9 beacon and the control scrambled beacon were as follows: 5'-NH2-TTCGCTGTGGTCCCGGCGTCCGGCCGGG-CGGGCGGGACCACAGCG-BHQ2-3', 5'-NH2-TCGCTGTTCCGCGACTCCACCTCCGTGGCGACAACCGTCGGAACAGCG-BHQ2-3', and 5'-NH2-TTCGCTGTGGTCCCGGCGTTTGCCCCCACAGCG-BHQ2-3', respectively. The carboxyl quantum dote (QD) nanoparticles (Life Technologies Inc., Eugene, CA, USA) were prepared as previously described [12] and then covalently conjugated to the hsv1-miR-
H18 and hsv2-miR-H9 beacons (10 pM) by incubation for 1 hour at room temperature. $\mathrm{N}$-ethyl- $\mathrm{N}^{\prime}$-dimethylaminopropyl carbodiimide was used to increase the coupling efficiency between the amine and carboxyl groups.

\section{Confocal Microscopy of $\mathrm{QD}_{565}$ Particles Within Tissues}

Tissues were examined by whole-mount immunocytochemistry as previously described [13]. The $\mathrm{QD}_{565}$-hsv1-miR-H18/hsv2miR-H9 beacons were used as follows for detection: $\mathrm{QD}_{565^{-}}$ hsv1-miR-H18 beacon and QD 255 -hsv2-miR-H9 beacons, and hsv-miR-HS beacon probes (1:1,000; Bioneer, Daejeon, Korea). Excitation was provided by a He/Ne laser (wavelengths, $405 \mathrm{~nm}$ and $543 \mathrm{~nm}$ ) and images of selected tissues were visualized using the $4 \times$ and $20 \times$ objectives of a scanning Zeiss LSM 710 confocal microscope (Carl Zeiss, Oberkochen, Germany) with an Axiovert $200 \mathrm{M}$. After scanning specific regions to determine the tissue distribution using the region of interest scan feature of Zen 2011 software (Carl Zeiss Microimaging GmbH, Jena, Germany), a virtual mask was drawn within the selected tissue area for a $5 \times 5$ tile scan area. Individual tissues were imaged using a $5 \times 5$ tile scan area with tile scan sections.

\section{Statistical Analyses}

Nonparametric tests were used to compare expression of the two miRNAs between groups, because data could not always be transformed to achieve normality. Receiver operating characteristic (ROC) curves were used to evaluate the difference in expression of the two miRNAs in cancer tissues and BPH control tissues. Spearman correlation coefficient (r) was used to evaluate the correlation between miRNAs in surrounding noncancerous tissue with that in matched CaP tissues. Statistical analyses was performed using IBM SPSS Statistics ver. 21.0 (IBM Co., Armonk, NY, USA), and $\mathrm{P}<0.05$ was considered statistically significant.

\section{RESULTS}

\section{Expression of hsv1-miR-H18 and hsv2-miR-H9-5p in CaP}

To investigate the potential association of two viral hsv1-miRH18 and hsv2-miR-H9-5p miRNAs with clinicopathological outcomes, the expression of these miRNAs in BPH tissue, cancer tissue, and noncancerous surrounding tissues was compared. Real-time PCR results revealed increased expression of hsv1-miR-H18 and hsv2-miR-H9-5p in CaP tissue compared to that in $\mathrm{BPH}$ tissue (each $\mathrm{P}<0.001$ ) (Fig. 2). To confirm the 

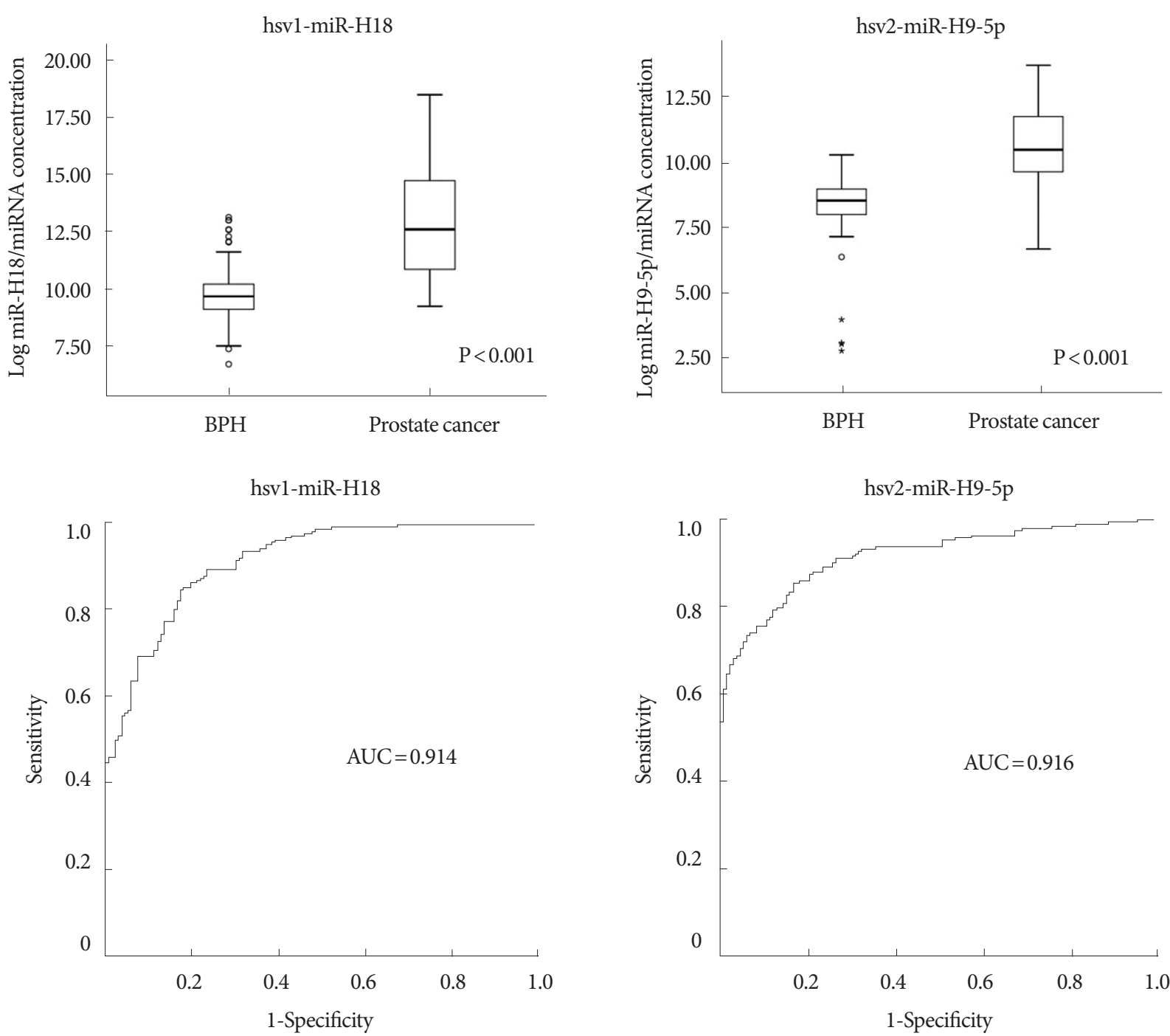

Fig. 2. Expression of hsv1-miR-H18 and hsv2-miR-H9-5p in benign prostatic hyperplasia (BPH) and prostate cancer (CaP) tissues. Expression of 2 microRNAs (miRNAs) was markedly higher in CaP tissues than in BPH tissues. The areas under the receiver operating characteristic (AUC) curves for hsv1-miR-H18 and hsv2-miR-H9-5p were 0.914 and 0.916, respectively.

specificity of the real-time PCR, PCR products were cloned into TA cloning vectors, and miRNAs from at least 20 clones were sequenced. Results showed 100\% identity between the hsv1miR-H18 and hsv2-miR-H9-5p clone, while no human homologs were detected in GenBank.

To determine whether this analysis might be used as a diagnostic tool, an ROC analysis was performed to assess how well hsv1-miR-H18 and hsv2-miR-H9-5p expression levels segregate patients with $\mathrm{CaP}$ from $\mathrm{BPH}$ controls. The areas under the ROC for hsv1-miR-H18 and hsv2-miR-H9-5p were 0.914 and 0.916 , respectively, indicating high sensitivity and specificity. There was no significant difference in the expression levels of the 2 miRNAs and or pathological characteristics between the groups, however (i.e., PSA $\leq 10 \mathrm{ng} / \mathrm{mL}$ vs. $>10 \mathrm{ng} / \mathrm{mL}$; Gleason score $\leq 7$ vs. $\geq 8$; and $\leq \mathrm{T} 3$ vs. T4 or metastasis) (Table 2). This indicates these miRNAs may be a useful tool to identify patients with $\mathrm{CaP}$, even though their expression levels do not correlate with disease severity.

\section{Expression of hsv1-miR-H18 and hsv2-miR-H9-5p in BPH Tissue, CaP Tissue, and Surrounding Noncancerous Tissue}

Both hsv1-miR-H18 and hsv2-miR-H9-5p were found to be expressed more highly in $\mathrm{CaP}$ tissue and surrounding noncancerous tissue than in $\mathrm{BPH}$ tissue (each $\mathrm{P}<0.001$ ) (Fig. 3). This 
Table 2. Expression of hsv1-miR-H18 and hsv2-miR-H9-5p in prostate cancer

\begin{tabular}{|c|c|c|c|c|c|}
\hline Variable & $\begin{array}{c}\text { No. of } \\
\text { patients }\end{array}$ & $\begin{array}{c}\text { hsv1-miR-H18 level } \\
\left(\times 10^{3} \text { copies/miRNA concentration }\right)\end{array}$ & P-value & $\begin{array}{c}\text { hsv2-miR-H9-5p level } \\
\left(\times 10^{3} \text { copies/miRNA concentration }\right)\end{array}$ & P-value \\
\hline Age (yr) & & & 0.495 & & 0.412 \\
\hline$<70$ & 101 & $280.4(58.8-4064.8)$ & & $38.3(14.5-136.8)$ & \\
\hline$\geq 70$ & 91 & $293.6(45.2-2043.7)$ & & $31.0(15.0-114.2)$ & \\
\hline PSA (ng/mL) & & & 0.465 & & 0.079 \\
\hline$<10$ & 78 & $355.0(53.5-3913.2)$ & & $49.8(17.6-142.0)$ & \\
\hline$\geq 10$ & 114 & $255.5(45.9-2374.4)$ & & $31.1(12.5-113.7)$ & \\
\hline Gleason score & & & 0.297 & & 0.195 \\
\hline$\leq 7$ & 103 & $295.0(62.8-4685.8)$ & & $39.3(16.3-136.3)$ & \\
\hline$\geq 8$ & 89 & $286.1(38.8-2429.6)$ & & $28.1(12.7-110.4)$ & \\
\hline Stage & & & 0.198 & & 0.241 \\
\hline $\mathrm{T} 3$ or less & 126 & $305.0(60.0-3290.7)$ & & $35.3(16.8-135.1)$ & \\
\hline T4 or metastatic & 66 & $257.0(35.0-1721.1)$ & & $30.4(11.2-104.2)$ & \\
\hline
\end{tabular}

Values are presented as median (interquartile range).

PSA, prostate-specific antigen.

The Mann-Whitney U-test was used to compare expression levels and clinical variables.
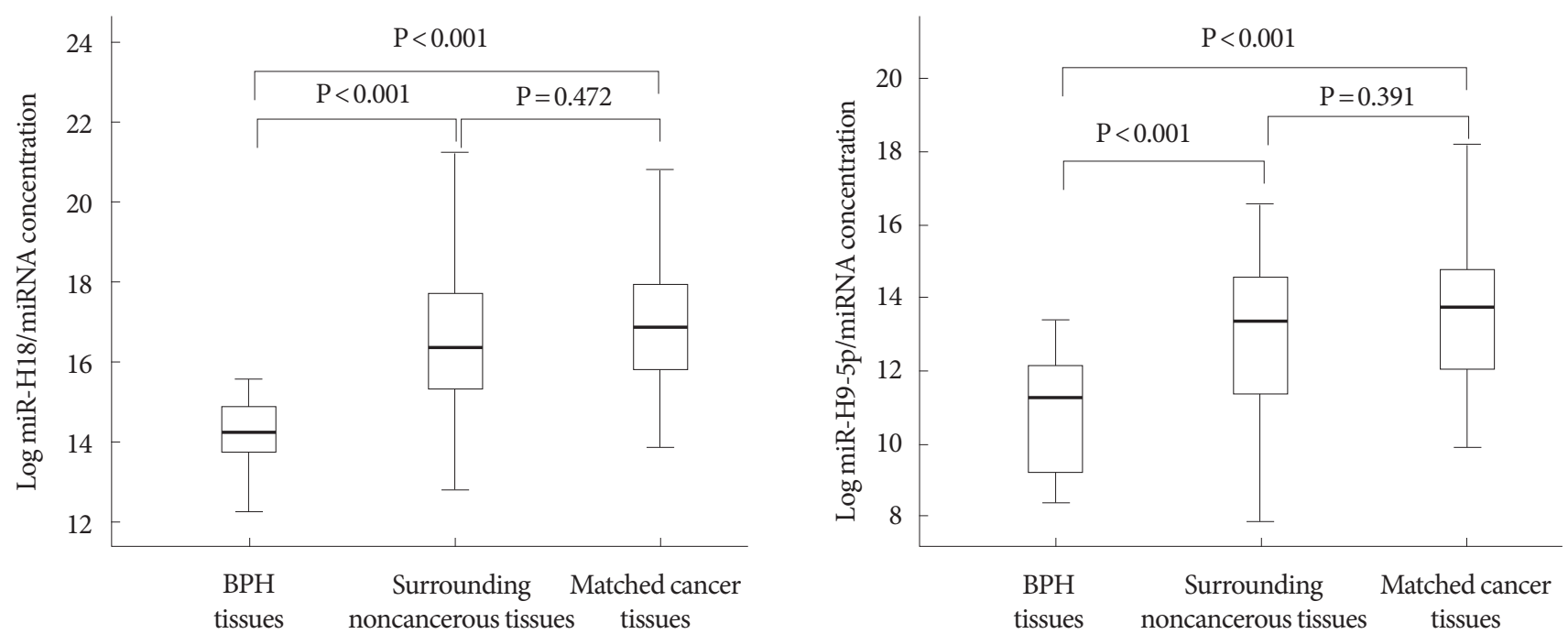

Fig. 3. Correlation between hsv1-miR-H18 and hsv2-miR-H9-5p in benign prostatic hyperplasia (BPH) tissue, prostate cancer (CaP) tissue, and matched, surrounding noncancerous tissue. Expression of the 2 microRNAs (miRNAs) was higher in CaP tissue and surrounding noncancerous tissue than in $\mathrm{BPH}$ tissue (each comparison, $\mathrm{P}<0.001$ ); however, there was no significant difference between the expression levels in surrounding noncancerous tissue and $\mathrm{CaP}$ tissue (each comparison, $\mathrm{P}>0.05$ ).

indicates that these miRNAs are highly expressed throughout the prostate when a tumor is present, and that their expression is lower in noncancerous prostate tissues. In addition, miRNA expression in surrounding noncancerous tissues significantly correlated with that of the matched CaP tissue (Spearman correlation $\mathrm{r}=0.317$ and $\mathrm{P}=0.044$ for hsv1-miR-H18, and $\mathrm{r}=0.759$ and $\mathrm{P}<0.001$ for hsv2-miR-H9-5p).

\section{Direct Staining of miRNAs in BPH, CaP, and Noncancerous Tissues}

A specific QD ${ }_{565}$-hsv1-miR-H18 and hsv2-miR-H9 beacon detection system was used to stain the two miRNAs in prostate 

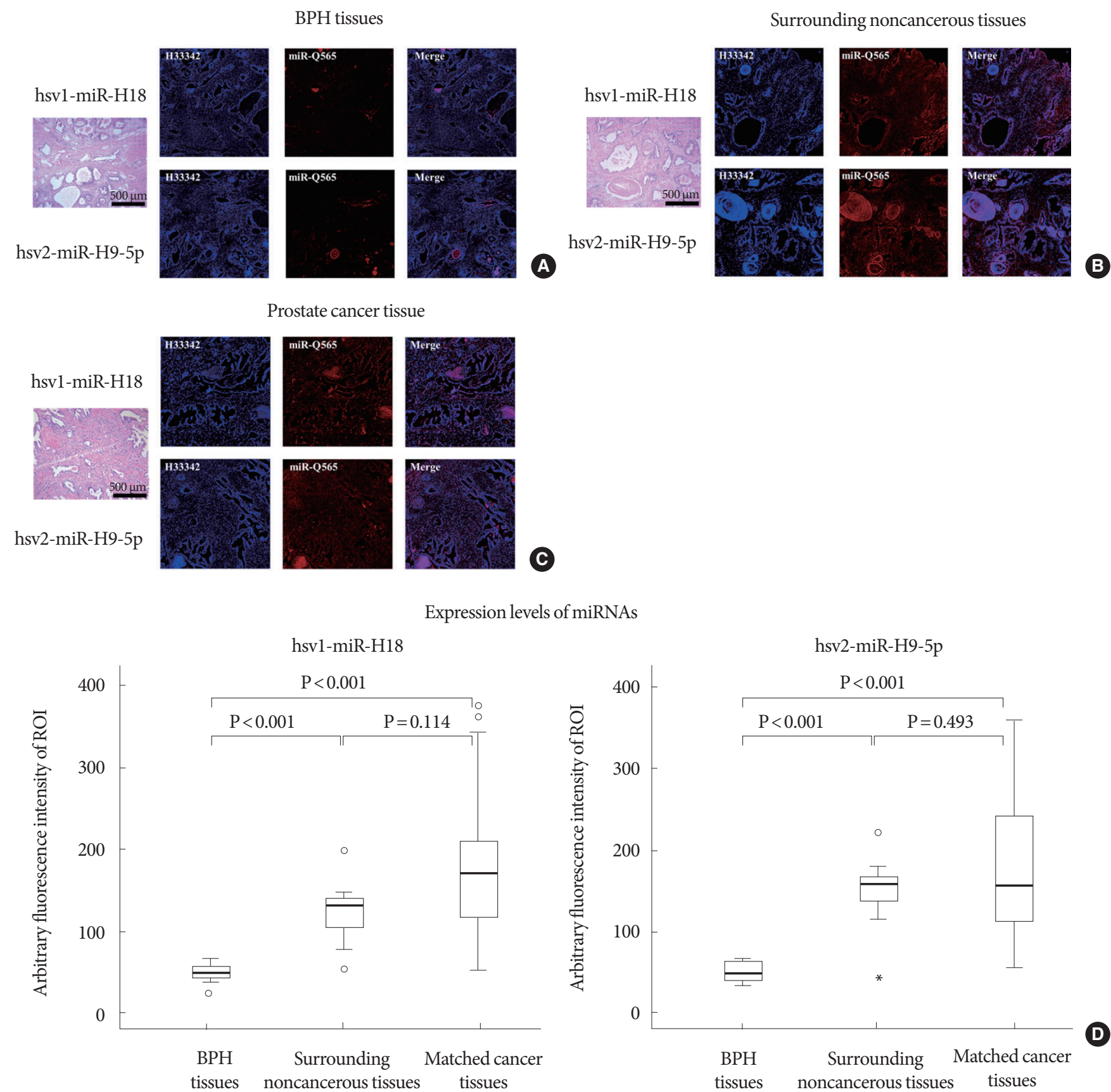
increased staining in the epithelial layer compared to that in the stroma. Interestingly, luminal cells, which have direct contact with seminal fluid, showed the strongest fluorescence intensity.

\section{DISCUSSION}

Here, we show that the expression levels of hsv1-miR-H18 and hsv2-miR-H95p were higher in $\mathrm{CaP}$ and surrounding noncancerous tissue than in BPH tissues. This suggests that these 2 miRNAs may be specific biomarkers for tumor-containing organs, as opposed to cancer-specific markers. These findings may be clinically important, suggesting it may be possible to detect early tumorigenesis before the cancerous tissue comprises a large portion of the prostate. The ability to diagnosis cancer while the disease is still localized within the originating organ has important implications, including increased likelihood of curative resection. Numerous previous studies have focused on the identification of cancer-specific biomarkers for CaP; however, when the disease is localized, the sensitivity and specificity of biomarker detection in body fluids is very low. PSA is a prostate-specific marker that can be detected in patient serum, and although it is considered an outstanding marker for detecting $\mathrm{CaP}$, it is not a cancer-specific marker, and so patients are often subjected to the painful transrectal biopsy procedure in order to rule out cancer. In this study, we evaluated two herpes virus-encoded miRNAs as organ-specific markers of cancer. We found that the expression levels of hsv1-miR-H18 and hsv2-miR-H9$5 \mathrm{p}$ were significantly higher in $\mathrm{CaP}$ tissue and the surrounding noncancerous tissue than in $\mathrm{BPH}$ tissue (each comparison, $\mathrm{P}<0.001$ ). Further, these results may explain why the miRNAs were easily detected in urine samples of patients with $\mathrm{CaP}[10]$.

Since the discovery of viral miRNAs in human tissues [14], many researchers have focused on identifying the functions and roles of these molecules in human cells; however, such research is still in its initial stages. While certain viral miRNAs are thought to enable viruses to evade the host immune system [8], to our knowledge, the direct relationship between virus-encoded miRNAs and initiation, progression, and metastasis of $\mathrm{CaP}$ has not been studied. Recent evidence suggests that $\mathrm{CaP}$ is associated with infection and inflammation caused by bacteria and viruses [3-5]. Therefore, it is possible that hsv1-miR-H18 and hsv2-miR-H9-5p play a role in CaP carcinogenesis, a finding that would be supported by our real-time PCR results. Future studies will be required to address this possibility.

One of the major risk factors for $\mathrm{CaP}$ is sexually transmitted infections (STIs). Given the solid evidence that human papillomavirus is the major causative agent of cervical cancer [15], it has been hypothesized that STIs such as those caused by Neisseria gonorrhoeae, Treponema pallidum, Chlamydia trachomatis, Trichomonas vaginalis, and herpes viruses may be associated with carcinogenesis of the prostate. While bacterial infections are also thought to cause inflammation and atrophy of the prostate, viral infections, and herpes viruses in particular, can lead to the transformation of prostate epithelial cells [16]. Several epidemiologic studies have suggested possible associations between STIs and $\mathrm{CaP}$ [17-19], but the data are inconsistent. A recent meta-analysis suggested that herpes virus type 2 infection is associated with an increased risk of $\mathrm{CaP}$ (odds ratio, 1.209; 95\% confidence interval [CI], 1.003-1.456) [20]. However, another meta-analysis showed that men who reported never having had an STI were at increased risk for CaP (summary relative risk [SRR], 1.49; 95\% CI, 1.19-1.92), and that men with gonorrheal infection also showed an increased risk of CaP (SRR, 1.20; 95\% CI, 1.05-1.37) [6]. However, no single STI, including herpes virus, has been definitively linked to CaP [6]. These conflicting findings may be a result of different methods used to detect antibodies against STI-causing agents in serum. Therefore, further well-designed investigations are needed to elucidate the potential links between STIs and increased risk of $\mathrm{CaP}$.

Because the prostate is not a target organ for herpes virus replication, it is possible that the presence of herpes virus-encoded miRNAs in CaP tissues and urine might not be linked to herpes infection of the prostate. However, given that there are no human homologs of either hsv1-miR-H18 or hsv2-miR-H9$5 p$, and that other herpes-encoded miRNAs (e.g., hsv1-miRH13, hsv2-miR-H2, and hsv2-miR-H7) are also enriched in $\mathrm{CaP}$ tissues (data not shown), it is difficult to rule out a direct association between herpes infection, with the subsequent presence of virus-encoded miRNAs, and CaP development. Therefore, the findings presented herein may provide the missing link between herpes virus infection and carcinogenesis of the prostate, and support the theory that STIs are a major risk factor for $\mathrm{CaP}$.

Our study had several inherent weaknesses. First, anatomical differences in the expression of the 2 virus-encoded miRNAs were not considered, even though $\mathrm{BPH}$ originates from the transitional zone, while $\mathrm{CaP}$ is usually from the peripheral zone. Second, we did not find any direct evidence of the source of these virus-encoded miRNAs. Further studies are needed to investigate the origin of these miRNAs. Lastly, because we did 
not perform an in vitro experiment, the influence of these miRNAs on CaP cells is uncertain.

In conclusion, there is increased expression of hsv1-miRH18 and hsv2-miR-H95p in CaP and surrounding noncancerous tissues compared to that in $\mathrm{BPH}$ tissues, suggesting that herpes virus-encoded miRNAs might be associated with tumorigenesis in the prostate. Further investigation will be required in order to elucidate the role of these miRNAs in relation to herpes viral infection as well as $\mathrm{CaP}$, and to confirm their validity as biomarkers.

\section{REFERENCES}

1. Jemal A, Siegel R, Xu J, Ward E. Cancer statistics, 2010. CA Cancer J Clin 2010;60:277-300.

2. Jung KW, Won YJ, Park S, Kong HJ, Sung J, Shin HR, et al. Cancer statistics in Korea: incidence, mortality and survival in 2005. J Korean Med Sci 2009;24:995-1003.

3. De Marzo AM, Platz EA, Sutcliffe S, Xu J, Gronberg H, Drake CG, et al. Inflammation in prostate carcinogenesis. Nat Rev Cancer 2007;7:256-69.

4. Sfanos KS, Isaacs WB, De Marzo AM. Infections and inflammation in prostate cancer. Am J Clin Exp Urol 2013;1:3-11.

5. Kwon OJ, Zhang L, Ittmann MM, Xin L. Prostatic inflammation enhances basal-to-luminal differentiation and accelerates initiation of prostate cancer with a basal cell origin. Proc Natl Acad Sci U S A 2014;111:E592-600.

6. Caini S, Gandini S, Dudas M, Bremer V, Severi E, Gherasim A. Sexually transmitted infections and prostate cancer risk: a systematic review and meta-analysis. Cancer Epidemiol 2014;38:329-38.

7. Pfeffer S, Sewer A, Lagos-Quintana M, Sheridan R, Sander C, Grässer FA, et al. Identification of microRNAs of the herpesvirus family. Nat Methods 2005;2:269-76.

8. Veksler-Lublinsky I, Shemer-Avni Y, Kedem K, Ziv-Ukelson M. Gene bi-targeting by viral and human miRNAs. BMC Bioinformatics 2010;11:249.

9. Kliche S, Nagel W, Kremmer E, Atzler C, Ege A, Knorr T, et al. Signaling by human herpesvirus 8 kaposin A through direct membrane recruitment of cytohesin-1. Mol Cell 2001;7:833-43.
10. Yun SJ, Jeong P, Kang HW, Kim YH, Kim EA, Yan C, et al. Urinary microRNAs of prostate cancer: virus-encoded hsv1-miRH18 and hsv2-miR-H9-5p could be valuable diagnostic markers. Int Neurourol J 2015;19:74-84.

11. Yoon TJ, Yu KN, Kim E, Kim JS, Kim BG, Yun SH, et al. Specific targeting, cell sorting, and bioimaging with smart magnetic silica core-shell nanomaterials. Small 2006;2:209-15.

12. So MK, Loening AM, Gambhir SS, Rao J. Creating self-illuminating quantum dot conjugates. Nat Protoc 2006;1:1160-4.

13. He S, Krens SG, Zhan H, Gong Z, Hogendoorn PC, Spaink HP, et al. A $\Delta$ Raf1-ER-inducible oncogenic zebrafish liver cell model identifies hepatocellular carcinoma signatures. J Pathol 2011;225: 19-28.

14. Pfeffer S, Zavolan M, Grasser FA, Chien M, Russo JJ, Ju J, et al. Identification of virus-encoded microRNAs. Science 2004;304:7346.

15. Bosch FX, Burchell AN, Schiffman M, Giuliano AR, de Sanjose S, Bruni L, et al. Epidemiology and natural history of human papillomavirus infections and type-specific implications in cervical neoplasia. Vaccine 2008;26 Suppl 10:K1-16.

16. Sutcliffe S. Sexually transmitted infections and risk of prostate cancer: review of historical and emerging hypotheses. Future Oncol 2010;6:1289-311.

17. Haid M, Sharon N. Immunofluorescent evidence of prior herpes simplex virus type- 2 infection in prostate carcinoma. Urology 1984;24:623-5.

18. Dennis LK, Coughlin JA, McKinnon BC, Wells TS, Gaydos CA, Hamsikova E, et al. Sexually transmitted infections and prostate cancer among men in the U.S. military. Cancer Epidemiol Biomarkers Prev 2009;18:2665-71.

19. Sutcliffe S, Giovannucci E, Gaydos CA, Viscidi RP, Jenkins FJ, Zenilman JM, et al. Plasma antibodies against Chlamydia trachomatis, human papillomavirus, and human herpesvirus type 8 in relation to prostate cancer: a prospective study. Cancer Epidemiol Biomarkers Prev 2007;16:1573-80

20. Ge X, Wang X, Shen P. Herpes simplex virus type 2 or human herpesvirus 8 infection and prostate cancer risk: a meta-analysis. Biomed Rep 2013;1:433-9. 